\title{
Perioperative considerations of hyperthyroidism in a patient with liver failure: case report
}

\author{
Byung Hwa Kim, Yun Hee Kim, Yoon Sook Lee, Woon Young Kim, Jae Hwan Kim \\ Department of Anesthesiology and Pain Medicine, Ansan Hospital, Korea University College of Medicine, Ansan, Korea
}

Received March 25, 2019

Revised May 13, 2019

Accepted May 14, 2019

Corresponding author

Jae Hwan Kim

Department of Anesthesiology and

Pain Medicine, Ansan Hospital,

Korea University College of

Medicine, 123, Jeokgeum-ro,

Danwon-gu, Ansan 15355, Korea

Tel: +82-31-412-5297

Fax: +82-31-412-5294

E-mail: anejhkim@korea.ac.kr

ORCID:

https://orcid.org/0000-0002-1360-1708
Hyperthyroidism is one of the most common endocrinologic disorders. When patients with hyperthyroidism undergo general anesthesia, potential perioperative complications such as atrial fibrillation, congestive heart failure, ischemic heart disease, thrombocytopenia may occur. Hyperthyroidism treatment is mainly divided into three parts: Medical treatment, radioactive iodine therapy, and surgical removal of the thyroid. However, the previous two treatments may not have a significant effect on patients with liver failure. In this report, we present a case where a patient with an underlying disease of uncontrolled hyperthyroidism and liver failure undergoing liver transplantation, where preoperative thyroid levels couldn't be managed aggressively due to liver failure. We managed hyperthyroidism using conservative management before and during surgery; perioperative use of steroids, beta-blockers, calcium channel-blockers, IV (Intravenous) fluids, body temperature regulation, opioid use such as remifentanil and fentanyl, sufficient sedation are all key in ensuring the hemodynamic stability of such a patient.

Keywords: Hyperthyroidism; Liver failure; Management

\section{INTRODUCTION}

Hyperthyroidism is one of the most common endocrinologic disorders [1-3]. It is present when thyroid hormone production is increased through various etiologies and can cause thyrotoxicosis, which is presented as high thyroid hormone levels. If uncontrolled, it can even lead to a state of multi-organ failure which we call 'thyroid storm' [4-6].

When patients with hyperthyroidism undergo general anesthesia, potential perioperative complications such as atrial fibrillation, congestive heart failure, ischemic heart disease, thrombocytopenia may occur [1,7]. Also, because thyroid hormones are glucuronidated and sulfated within the liver and subsequently excreted into bile, liver dysfunction is commonly observed in patients with thyroid disease
$[8,9]$. This is why all patients with an underlying thyroid condition are recommended to take a preoperative thyroid function test. If found abnormal, it is then advised to correct any abnormal levels before the scheduled operation [1,7].

Hyperthyroidism treatment is mainly divided into three parts: Medical treatment, radioactive iodine therapy, and surgical removal of the thyroid [1-3,5]. In medically treated patients, the drugs that are used (PTU, methimazole) have a potential to cause liver injury and greater consideration is taken with patients with low hepatic reserves $[2,5,6,10]$. Also, the onset of these drugs is 6 to 8 weeks [1,5-7], making it ineffective in controlling the disease before an emergency operation. Although delaying surgery until thyroid levels normalize may be optimal, this is not always the case in emergency situations. Anesthesiologists and surgeons will 
ultimately have to weigh the risk and benefits, and there will be cases where a patient with high thyroid levels requires general anesthesia.

In this report, we present a case where a patient with an underlying disease of uncontrolled hyperthyroidism and liver failure undergoing liver transplantation, where preoperative thyroid levels couldn't be managed aggressively due to liver failure.

\section{CASE REPORT}

The patient was a 46 year old male with an underlying disease of Hepatitis B and Graves' disease, and was treated with antiviral and anti-thyroid medication. However, the patient withheld all medication 7 months prior to admittance to the hospital. He was first admitted for a sudden onset of jaundice and was diagnosed with acute liver failure, but was discharged on his own volition. After 3 days of being discharged, he was readmitted through the emergency room due to his sudden drowsy mentality.

In the initial laboratory tests, liver function tests (AST/ALT) were 3,266/2,738 IU/L, total bilirubin was $38.50 \mathrm{mg} / \mathrm{dL}$, prothrombin time was $7 \%$, prothrombin time international normalized ratio was 9.23 , activated partial thromboplastin time was $78.7 \mathrm{sec}$, Albumin was $3.6 \mathrm{~g} / \mathrm{dL}$, HBV-DNA titer was $>170,000,000 \mathrm{IU} / \mathrm{mL}$, BUN/Cr were 4.8/0.91 mg/dL, electrolytes were normal (Sodium was $136 \mathrm{mmol} / \mathrm{L}$, potassium was $4.8 \mathrm{mmol} / \mathrm{L}$, and chloride was $98 \mathrm{mmol} / \mathrm{L}$ ), and no abnormalities were seen in the urinalysis. Preoperative evaluation revealed a normal brain CT, while an echocardiogram showed no regional wall motion abnormalities, a normal ejection fraction of 55-60\% and pulmonary artery pressure of $31 \mathrm{mmHg}$. The patient was diagnosed with acute exacerbation of chronic viral liver disease, hepatic encephalopathy, and with a MELD score of 45 , was eligible for liver transplantation.

Initial thyroid function tests were extremely high (fT $4>7.77$ ng/dL, TSH $0.060 \mathrm{uIU} / \mathrm{mL}$, T3 $139.1 \mathrm{ng} / \mathrm{dL}$ ). The vital signs of the patient during intensive care unit (ICU) care showed blood pressure of 200-120/100-60 mmHg, heart rate of 110-150 bpm, daily fever of 38 degrees celsius. This was managed by beta-blockers, antibiotics and antipyretics, whilst the patient was sedated through continuous midazolam and fentanyl infusion. Vital signs were kept at blood pressure 140-110/80-60 mmHg, heart rate 120-140 bpm while the patient waited for liver transplantation.

The patient presented to the operation room with blood pressure at 136/72 $\mathrm{mmHg}$, heart rate $136 \mathrm{bpm}, \mathrm{SpO}_{2}$ (saturation of percutaneous oxygen) $98 \%$ without premedication. Electrocardiogram showed a normal sinus rhythm, body temperature 36.4 degrees celcius, and he was sedated. Intraoperative monitoring included electrocardiography, heart rate, blood pressure, $\mathrm{SpO}_{2}, \mathrm{EtCO}_{2}$ (end tidal $\mathrm{CO}_{2}$ ), invasive monitoring such as arterial cannulation of the femoral and radial arteries, and central cannulation of the femoral and internal jugular vein was done for rapid fluid therapy. A large-bore catheter was placed in the left internal jugular vein. Induction of anesthesia was achieved using desflurane inhalation and $50 \mathrm{mg}$ of IV rocuronium, maintenance of anesthesia was done using $\mathrm{O}_{2} 1.1 \mathrm{~L} / \mathrm{min}$, fresh air $1.9 \mathrm{~L} / \mathrm{min}$, and desflurane at 3-8 vol\%.

15 minutes after the initiation of surgery, blood pressure was 120/60 mmHg, heart rate $130 \mathrm{bpm}$ and esmolol infusion and fentanyl $50 \mathrm{mcg}$ bolus was given twice. Afterwards blood pressure was controlled between 120-90/60-50 $\mathrm{mmHg}$, heart rate at $110 \mathrm{bpm}$. To keep the patient at normothermic levels, various ways, such as cool/warm blanket, hot line, and air warmer, were used throughout the surgery and the patient's body temperature ranged from 35.7 to 36.8 degrees celcius.

After reperfusion, blood pressure rose to $150 / 60 \mathrm{mmHg}$, and heart rate rose to $130 \mathrm{bpm}$. Esmolol infusion dose was increased to control this change. After the surgery, dexmedetomidine (Precedex) was infused while the patient was transported to the ICU.

In order to control the heart rate, esmolol infusion was maintained postoperatively. At POD (postoperative day) \#4 the patient was extubated and at POD \#5 bisoprolol (oral $\beta$-blocker) was given instead and esmolol infusion was discontinued. At POD \#9 the patient was moved to the general ward. Postoperative fT4 levels at POD \#7 was $2.3 \mathrm{ng} / \mathrm{dL}$ but quickly rose again to $3-4 \mathrm{ng} / \mathrm{dL}$, and TSH was $0.05 \mathrm{uIU} / \mathrm{L}$ at POD \#7 and rose to $0.01 \mathrm{uIU} / \mathrm{L}$ afterwards. Although methimazole was primarily considered in treating the underlying hyperthyroidism, liver function was not fully normal after liver transplantation. Steroid (continued until POD \#12), and bisoprolol was maintained until liver function returned to normal levels at POD \#26. The patient was discharged 30 
days after having liver transplantation surgery.

\section{DISCUSSION}

The patient in this case was diagnosed with Graves' disease but discontinued his antithyroid medication and was at a uncontrolled hyperthyroidism state at the time of his liver transplantation surgery. The reason for this patient's liver failure wasn't deemed to be caused by a thyroid storm or the adverse effects from antithyroid medication. This was because of several facts; he discontinued his antithyroid medication 7 months before the diagnosis, liver function was normal 3 days before the onset of jaundice, and his HBV-DNA titer was very high at $>170,000,000 \mathrm{IU} / \mathrm{mL}$ because he had also discontinued his HBV medication. It was thought that the patient's chronic HBV infection caused the sudden liver failure.

Managing hyperthyroidism is very important because it causes many cardiac conditions but also can cause liver injury. Because thyroid hormones are glucuronidated and sulfated within the liver and subsequently excreted into bile, liver dysfunction is commonly observed in patients with thyroid disease. The reported prevalence of liver dysfunction in patients with hyperthyroidism varies widely, ranging from $15 \%$ to $79 \%[8,9]$. For this patient, he had hepatic encephalopathy as a result of severe liver failure and a MELD score of 45.

Prior to the liver transplantation, many considerations were made in managing the patient's thyroid function. Antithyroid medication was first considered but quickly dismissed due to the probability of liver toxicity $[1,2,5,7,11]$. Generally, antithyroid medication (PTU, methimazole) induced liver toxicity are reported to be under $0.5 \%[2,11]$. Especially, PTU is reported to even cause liver failure [2]. Methimazole causes liver injury in a cholestatic pattern and PTU in a hepatic pattern [11]. Also, it generally takes 6 to 8 weeks for the maximal effect of the drug and therefore was another reason for oral medication not being an optimal method of hyperthyroidism management for this case [1,5-7]. Lugol's solution had to be used after antithyroid medication $[6,7,12]$, so it wasn't applicable to this patient. Radioactive iodine is widely used in patients with hepatic dysfunction, but there are cases which report hepatotoxicity and exacerbation of liver function so it must be used with care [3,13-15]. Also, the effects of radioactive iodine takes weeks to assess so it is not fit for use in emergency situations $[3,5,6$. Glucocorticoid was also considered due to its effects of reducing the conversion of thyroxine to triiodothyronine within 24 hours of usage, however it is only effective when used concomitantly with other therapies [7,12,16].

Therefore, The preoperative management of hyperthyroidism was made difficult due to the patient's liver condition, and we had to resort to conservative and symptomatic treatment, such as beta-blockers, hydration and body temperature control, in order to proceed with the surgery $[5,7]$. After the surgery, glucocorticoid was continuously used, and methimazole was given after liver function normalized.

It is recommended that all patients with hyperthyroidism have their thyroid function levels corrected before general anesthesia, due to the numerous perioperative complications such as atrial fibrillation, congestive heart failure, ischemic heart disease, thrombocytopenia, and liver dysfunction might arise during surgery. This may be done using antithyroid medication, radioactive iodine, and surgical resection of the thyroid tissue. However, antithyroid medication potentially causes liver injury and Lugol's solution is only effective after the use of antithyroid medication so it can't be considered in cases of liver failure. In this case, the patient had severe liver injury and thus couldn't have delayed his liver transplantation in order to control his thyroid function. Therefore, an approach using conservative treatment must be taken in order to manage hyperthyroidism before and during surgery; perioperative use of steroids, beta-blockers, calcium channel-blockers, IV fluids, body temperature regulation, opioid use such as remifentanil and fentanyl, sufficient sedation are all key in ensuring the hemodynamic stability of such a patient. Whenever possible, postoperative use of antithyroid medication must ensue to treat hyperthyroidism after the liver function normalizes again.

\section{CONFLICT OF INTEREST}

No potential conflict of interest relevant to this article was reported.

\section{REFERENCES}

1. Farling PA. Thyroid disease. Br J Anaesth 2000;85:15-28. 
2. Wang MT, Lee WJ, Huang TY, Chu CL, Hsieh CH. Antithyroid drug-related hepatotoxicity in hyperthyroidism patients: a population-based cohort study. Br J Clin Pharmacol 2014;78:619-29.

3. Wang R, Tan J, Zhang G, Zheng W, Li C. Risk factors of hepatic dysfunction in patients with Graves' hyperthyroidism and the efficacy of 131 iodine treatment. Medicine (Baltimore) 2017;96:e6035.

4. Calissendorff J, Falhammar H. Rescue pre-operative treatment with Lugol's solution in uncontrolled Graves' disease. Endocr Connect 2017;6:200-5.

5. Vaidya B, Pearce SH. Diagnosis and management of thyrotoxicosis. BMJ 2014;349:g5128.

6. Gilbert J. Thyrotoxicosis - investigation and management. Clin Med (Lond) 2017;17:274-7.

7. Palace MR. Perioperative management of thyroid dysfunction. Health Serv Insights 2017;10:1178632916689677.

8. Khemichian S, Fong TL. Hepatic dysfunction in hyperthyroidism. Gastroenterol Hepatol (N Y) 2011;7:337-9.

9. Lin TY, Shekar AO, Li N, Yeh MW, Saab S, Wilson M, et al. Incidence of abnormal liver biochemical tests in hyperthyroidism. Clin Endocrinol (Oxf) 2017;86:755-9.

10. Heidari R, Niknahad H, Jamshidzadeh A, Eghbal MA, Abdoli
N. An overview on the proposed mechanisms of antithyroid drugs-induced liver injury. Adv Pharm Bull 2015;5:1-11.

11. Papachristos DA, Huynh J, Grossman M, MacIsaac RJ. Liver dysfunction and anti-thyroid therapy. SAGE Open Med Case Rep 2015;3:2050313X14568335.

12. Satoh T, Isozaki O, Suzuki A, Wakino S, Iburi T, Tsuboi K, et al. 2016 Guidelines for the management of thyroid storm from The Japan Thyroid Association and Japan Endocrine Society (First edition). Endocr J 2016;63:1025-64.

13. Fard-Esfahani A, Emami-Ardekani A, Fallahi B, Fard-Esfahani P, Beiki D, Hassanzadeh-Rad A, et al. Adverse effects of radioactive iodine-131 treatment for differentiated thyroid carcinoma. Nucl Med Commun 2014;35:808-17.

14. Kim CW, Park JS, Oh SH, Park JH, Shim HI, Yoon JW, et al. Drug-induced liver injury caused by iodine-131. Clin Mol Hepatol 2016;22:272-5.

15. Jhummon NP, Tohooloo B, Qu S. Iodine-131 induced hepatotoxicity in previously healthy patients with Grave's disease. Thyroid Res 2013;6:4.

16. Suwansaksri N, Preechasuk L, Kunavisarut T. Nonthionamide drugs for the treatment of hyperthyroidism: from present to future. Int J Endocrinol 2018;2018:5794054. 